EPiC Series in Education Science
Volume 1, 2017, Pages 62-69

\title{
An Ethnographic Investigation into a Series of Incidents on a large Construction Project
}

\author{
David Oswald \\ RMIT University, Australia \\ david.oswaldermit.edu.au
}

\begin{abstract}
A series of incidents in a short period created cause for concern on a large construction project in the UK $(+£ 500 \mathrm{~m})$. Incident investigations are one of the ways to learn about safety failings, so that remedial action can be put into place to avoid a recurrence. The researcher was a member of the $H \& S$ department, with the role of a participant observer during the incident investigation period. Data collection included: informal conversations with employees; attending safety and accident investigation meetings; viewing project documents; and attending the safety stand down that occurred. The case study findings revealed that a blame culture restricted information flow on the incidents; and consequently there was a focus on easily observable unsafe acts, and static unsafe conditions, providing a narrow rather than deep perspective. These acts and conditions, such as a lack of compliance with PPE, or a weather condition, were often difficult to manage. For safety understanding the project repeatedly used Heinrich's (1931) seminal work as a foundation. However, this work is arguably outdated as it focuses on accidents on an individual rather than complex socio-technical level.
\end{abstract}

Keywords: construction, safety, accident investigations, ethnographic methods

\section{Introduction}

Learning from previous accidents and incidents is important for preventing future injuries and losses. Hence even the most trivial incidents and near misses should be fully investigated, as there are potential learning opportunities without having to endure the consequences of an accident. During a ten-day period there were eight incidents on a large construction project in the UK $(+£ 500 \mathrm{~m})$. The researcher spent time as a member of the H\&S department during this period, and explored the investigation process for these accidents. The main aim of the research was to understand the greatest challenge to undertaking effective incident investigations. 


\section{Incident investigations}

In early safety work, Heinrich's (1931) focused on safety at the individual worker level, highlighting that $88 \%$ of accidents were caused by man failings. However, it has since been argued that this view on the cause of accidents is not compatible with the modern perspective; and that current thinking about how accidents occur and their casual factors has moved away from individual worker behavior, with emphasize being placed on improving the work system (Manuele, 2011). Depending on an organization's perspective, the accident investigation could reveal very different causes; and investigations that do not relate to the actual causal factors, will inevitably have corrective actions that are misdirected and ineffective (ibid).

An event investigation is the collection and examination of facts related to an occurred specific event (Harms-Ringdahl, 2004). Incidents are events that could have or have resulted in harm, such as a near miss, minor or major accident. As such, incident investigations incorporate events that could be accidents, often referred to as a 'near miss' or 'close call', as well as accidents. An accident investigation is one of the elements in the process of learning from accidents (Cedergren, 2013). Fully investigating accidents and incidents can reveal information that can be instrumental in avoiding the recurrence of subsequent failures (Johnson \& Holloway, 2003). For safety improvements it is essential that the recommendations on remedial actions presented in these investigations are implemented (Cedergren, 2013). Kletz (2002) highlighted that missed opportunities following an accident investigation include:

- Only finding one cause, usually the final trigger before the accident

- A focus on immediate causes but not ways of avoiding weaknesses in management

- Using the broad term human error without being more specific

- A focus on causes that little can be done about

- An emphasis on procedural change rather than design

- Experience learnt is not shared with others

- Lessons learned are forgotten and the accident reoccurs

Kletz (2006) also highlighted that the report deriving from the investigation should not be viewed as 'closing out' a problem, but instead ask 'what else could be done'? Near misses, minor accidents and some major accidents have investigations that are typically undertaken by the construction organizations where the incidents occurred. Severe accidents are sometimes investigated by government bodies, such as the HSE (Health \& Safety Executive) in the UK, which satisfies both: the public need for answers to what happened; and their need for reassurance that action will be taken to address the failings that led to the accident (Boraiko et al., 2008). The seriousness of a major accident and the potential legal implications can result in a more narrow and closed investigation, as opposed to near misses and minor accidents, which can be more open during the investigation process.

\section{Research methods}

Ethnography is a method of observing specific group in their natural setting (Phelps and Horman, 2010). Proponents of the use of ethnographic approaches in safety research have claimed that it can: offer rich and practical understanding concerning the complexity of informality in safety learning processes (Baarts, 2009); and enable a more sophisticated and nuanced assessment of safety culture than possible through other assessment techniques (Strauch, 2015). Pink et al. (2010) also argued that ethnographic approaches can go beyond what could be said in an interview; and Oswald et al. (2014a) 
explained that by establishing rapport with participants, the researcher can get access to data that may not normally be available, such as insights gathered from an accident investigation.

Ethnographic research is it often very time-consuming in comparison to other methods; and with regard to accident investigations, Strauch (2015) pointed out that ethnographic methods require more time to conduct a study than is reasonably available to incident investigators. Research adopting ethnographic approaches to explore accident investigations are rare, though there have been a few studies. For instance, Sanne (2008) found that in an incident-reporting scheme, employees must be given ownership, must know how and why to use it, and that there is a need feedback on root causes; while Oswald et al. (2015) revealed that an accident investigation found a combination of: time pressure; the shortage of skilled-labor; untrained workers; and poor planning all contributed to a minor accident.

As part of a wider three year ethnographic study, the researcher visited the construction project between one and three times a week for three years, adopting the role as a participant observer, who had no control over events or investigation processes. This case study focuses on the ten day period where eight incidents occurred on a large construction project $(+£ 500 \mathrm{~m})$ in the UK. On this project participant observation included a mixture of: attending accident investigation meetings, H\&S meetings, site offices, canteens, work sites, the safety stand down, viewing project documents, and having informal discussion with employees throughout the hierarchy from directors to labourers. The data collection and analysis were a concurrent process (Silverman, 2013), and was collected and analysed until a point of saturation was reached (Kumar, 2005).

\section{Discussion of findings}

H\&S manager: 'I don't know what the hell is going on. Another incident today. Get out there, get talking to people, let's find out what is going on'

On the return from the Christmas break period, a series of incidents were a cause for concern on a large construction project. Investigations were carried out, but gathering all the relevant information was challenging. One of the accidents occurred when a welder dropped his chip hammer on the level below; and when he went to retrieve his hammer, he then fell through a partially unprotected temporary opening, suffering minor injuries. In the investigation, the H\&S team had doubts about whether they were receiving all the information. One H\&S advisor stated:

'Call me a skeptic but I went down there, and you can see the outline from the dust and cuttings of where the baton [wooden plank] was. His [injured persons] story of what happened doesn't add up with the evidence. He's not thrown himself down there, but there is something not right. I don't think we have all the details, and we probably never will.'

When incidents occurred, the H\&S team were frustrated they were often not able to gather all the relevant information. H\&S professionals would frequently make remarks such as:

'We are being fed bullshit. Information is being sanitized at every level.'

With incidents that could lead to disciplinary action, information was particularly restricted; and where gross misconduct occurred, it was recognized that individuals should be held accountable. However where acts, such as mistakes or errors occur, there should a perception that reporting can 
follow without fear of blame (Johnson \& Holloway, 2003; Reason, 2008). At least in the UK, it is more legally convenient to blame individuals (Reason, 2008); and investigating employee-based causes can be attractive to internal investigators, as they may be put in a difficult position if an underlying cause of an incident is an organization's policy or culture (Kletz, 2006). However such a blame culture can create an environment that is very difficult to learn from (Reason, 2008).

The lack of information meant that the causes of the incidents were often attributed to unsafe conditions, rather than unsafe acts. Smith et al. (2017) noted that it is more difficult to observe fluid and momentary unsafe acts when compared to static and unchanging unsafe conditions. The acts that were documented were typically 'easy to observe' acts (see Fleming and Lardner, 2002), such as not wearing the correct PPE (personal protective equipment). This unsafe act is typically observable for a longer period than other fluid and momentary unsafe acts. Hence there tended to be a focus on easily observable unsafe acts (such as lack of PPE) and static unsafe conditions as causes. For instance, one minor first aid accident occurred when a delivery driver was not wearing gloves and cut his hand. The lessons learned document stated:

'Enforce offloading procedure, incorrect PPE for the task'

H\&S advisors were continually frustrated with the breaking of site rules such as non-compliance with PPE. Walker (2010) explained that the workers had developed a 'counterculture' to the official safety pronouncements of management. One of the greatest challenges the H\&S department faced was compliance with tool-tethers to avoid dropped tools, as this posed a high-risk. Incidents where tools were dropped raised the following documented lessons learned:

'Tool lanyards should be used where risk exists. Exclusion area below to be enforced.'

However even when risks did exist and tethers should have be worn, this site rule was not always adhered to. Workers explained that sometimes the tethers available were impractical, depending on the motion of the tool (e.g. twisting), while other workers simply did not want their own tools permanently tethered. One operative stated:

'We are the guys having to use the tethers, but we weren't consulted. They just buy a bunch and expect us to use them, when they might not work for all us. We have scaffs, joiners, welders, all doing different work.'

The workers did not believe the tethers were practical for all trades and scenarios, but superiors argued it was a site rule, and therefore must be adhered to. Paap (2003) proposed that in the construction industry safety should be interpreted in two forms: the official procedures and the actual working operations - a distinction that represents the difference between the rules stated and the rules that actually govern the workplace. This double-provision was described by Paap (2003:221) as 'a Bait-and-switch, since it clearly serves to advantage the employers at the expense of the workers'. This was a contentious issue as workers desired more 'common sense' with rules.

While there were often issues surrounding the PPE compliance as an unsafe act; unsafe conditions were more frequently highlighted as incident causes. For instance, a trip and fall resulting in a minor injury led to a corrective action documented as:

'Control of access to incomplete walkways, correct protection and marking of openings' 
An Ethnographic Investigation into a Series of Incidents on a Large Construction Project D. Oswald

Most of the unsafe conditions the contractors had control over; but others they had little control over. One of these conditions was the wind, which is an accident investigation finding that Kletz (2002) would classify as a missed opportunity, as very little can be done about it. A minor first aid accident that occurred on site was logged as:

\section{'Dust/grit blown in eye, eye rinsed out'}

The lessons learned from this event were detailed: 'High Winds- continuous reassessment of tasks/prevailing weather conditions'. Rozenfeld et al. (2010) highlighted that exposure to weather conditions is one of the many unique characteristics of the construction industry. The weather is not just a condition that can be unsafe, but it also disrupts the work. As work falls behind schedule production pressures can increase; which has been linked to poorer safety performance (Oswald et al., 2013; Han et al., 2014). Following an incident where an operative tripped, fell over and required first aid, an H\&S stated:

'We are 8 days behind already because of the weather. Guys are getting frustrated that they are behind, and are taking risks by not concentrating or paying attention'

Previous work has suggested there is a particularly the high risk tolerance found on construction sites (e.g. Rawlinson and Farrell, 2009). Oswald et al. (2014b) pointed out that as risks in the construction industry are mostly voluntary, under personal control, non-dread and known, and therefore they are more likely to be accepted and under-rated. The H\&S advisors believed that production pressures caused by weather disruptions were initiating risk-taking, as workers were focusing on job completion, rather than taking the time to finish the task safely. The Project Director reinforced this during the safety stand down:

'There is no doubt we are under significant production pressure, but nothing is more important than safety. Take care when you are out there. I know some of you don't go out there often, but keep aware. Any of us can trip, fall or injure ourselves ... it seems trivial... but it happens.'

Slips, trips and falls are common accidents that are often have minor rather than major severity. It is arguable that some of these slip, trip and fall accidents are unavoidable or inevitable. However, the project management team believed the frequency of them was in part due to the production pressure and risk-taking behavior. The concerning accident trend initiated a safety stand down to raise awareness, but this was not as successful as hoped:

Commercial Director: 'It is amazingly to think of all the things we do with safety, systems we have in place, number of safety professionals, safety training, PPE and even initiatives like the stand down... and still... and still... it didn't seem to help because the day after the stand down finished we had another incident.'

The H\&S team reflected on the safety stand down and concluded that there were a wide range of differences in presentation quality. Those that were poorly communicated frustrated the H\&S professionals:

$\mathrm{H} \& \mathrm{~S}$ advisor: 'there were poor presentations; presentations with no open questions at the end to involve the guys [workers], and one had work going on next door so you could hardly hear. These presentations should come from the managers to show their commitment, but some aren't delivering it the way it should be.' 
An Ethnographic Investigation into a Series of Incidents on a Large Construction Project D. Oswald

Within the safety stand down document distributed the incident rate was described as:

' $a$ worrying trend which directly relates to the accident triangle which we are all accustomed to seeing. In reality the numbers within these accident triangles ARE PEOPLE!'

In early safety research, Heinrich (1931) proposed that there was relationship between near misses, minor incidents, and major accidents; but its use has been criticized in the modern era (Manuele, 2011). At the time, Heinrich's work was ground-breaking, but its appropriateness within modern complex socio-technical systems has been brought into question. The accident triangle focuses on individual unsafe acts and personal injury accidents. Reason (2008) explains that this is an intuitively appealing approach which is frequently, but also inappropriately applied to organizational accidents. This is partly because accidents usually occur from multiple and interacting causal factors that often have organizational, cultural, technical or operational systems origins. Despite this, the large construction project repeatedly referred to Heinrich's work for guidance, such as in the safety stand down document. One H\&S advisor explained:

'We are still using research from the 1930s. There must be more recent work. There is a gap there because the research knowledge is not getting transferred. When I look at the recent research, it's so complex, all these models, what does it even mean?'

The practitioners acknowledged they struggled to understand some of the more recent safety research, such as the socio-technical accident models developed. While important research developments have encompassed the complexity of accidents, to have practical use in the industry, such models need to be understood in the field. In comparison, Heinrich's accident triangle is easily understood, and therefore frequently used, despite being arguably out-dated.

\section{Conclusions}

Incident investigations have the potential to reveal important safety information to avoid future accidents. It was found that the greatest challenge to having an effective incident investigation was successfully gathering all the information on the incident. Important information was often not disclosed by workers, due to fears of blame. The restricted information meant there was a focus on easily observable unsafe acts, and static unsafe conditions, which provided a narrow, rather than deep perspective. Through this perspective, recurrent incidents were difficult to solve, as they often involved conditions that were hard to control (e.g. weather) or unsafe acts that were frequently undertaken and challenging to change (e.g. a lack of PPE). The safety stand down undertaken to raise awareness was ineffective due to varying levels of presentation quality across the site. It is recommended that there is a considerable effort to create a no-blame culture within organizations.

Heinrich's (1931) work focused on safety at the individual worker level, yet this is not aligned with current thinking, with emphasize being placed on the work system. In this case study, practitioners frequently referred to Heinrich's work for safety understanding, which suggests there may be a gap between modern safety research and the practical needs of the industry. The case study has raised the question of how modern research work can encompass the complexity of accidents, but also be understandable for practitioners, to have greater impact in the construction industry. 
An Ethnographic Investigation into a Series of Incidents on a Large Construction Project D. Oswald

\section{References}

Baarts, C. (2009). Collective individualism: the informal and emergent dynamics of practising safety in a high-risk work environment. Construction Management and Economics, 27(10), 949-957.

Boraiko, C., Beardsley, T., \& Wright, E. (2008). Accident Investigations One Element of an Effective Safety Culture. Professional Safety, 53(09).

Cedergren, A. (2013). Implementing recommendations from accident investigations: a case study of inter-organisational challenges. Accident Analysis \& Prevention, 53, 133-141

Fleming, M., \& Lardner, R. (2002). Strategies to promote safe behaviour as part of a health and safety management system. Research report 430

Han, S., Saba, F., Lee, S., Mohamed, Y., \& Peña-Mora, F. (2014). Toward an understanding of the impact of production pressure on safety performance in construction operations. Accident Analysis \& Prevention, 68, 106-116.

Harms-Ringdahl, L. (2004). Relationships between accident investigations, risk analysis, and safety management. Journal of Hazardous materials, 111(1), 13-19.

Heinrich, H. W. (1931). Industrial Accident Prevention. New York: McGraw-Hill

Johnson, C. \& Holloway, C.M. (2003) A survey of logic formalisms to support mishap analysis, Reliability Engineering and System Safety, 80 (3) pp. 271-291

Kletz, T.A. (2002), Accident investigation - missed opportunities, Process Safety and Environmental Protection, 80 (B1) (2002), pp. 3-8

Kletz, T. (2006). Accident investigation: keep asking “why?” Journal of Hazardous Materials, 130(1-2), 69-75.

Kumar, R. (2005) Research Methodology. 2nd edn. London: Sage Publications Limited

Manuele, F. A. (2011). Reviewing Heinrich: Dislodging two myths from the practice of safety. Professional Safety, 56(10), 52.

Oswald, D., Sherratt, F., Smith, S. (2013) Exploring factors affecting unsafe behaviours in construction, In Smith, S D (Ed.) and Ahiaga-Dagbui, D D (Ed.), Proceedings of the 29th Annual ARCOM Conference, Association of Researchers in Construction Management, 335-344

Oswald, D., Sherratt, F., \& Smith, S. (2014a). Handling the Hawthorne effect: The challenges surrounding a participant observer. Review of Social Studies, 1(1), 53-73.

Oswald, D., Sherratt, F., \& Smith, S. (2014b). Risk Perception and Safety Behaviour: An Ethnographic Study. Proc. CIB W099 Achieving Sustainable Construction Health and Safety, 2-13

Oswald, D., Smith, S., \& Sherratt, F. (2015). Accident investigation on a large construction project: An ethnographic case study. Procedia Manufacturing, 3, 1788-1795. 
An Ethnographic Investigation into a Series of Incidents on a Large Construction Project D. Oswald

Paap, K. (2003). Voluntarily put themselves in harm's way: the 'bait and switch' of safety training in the construction industry. In D. Bills (Ed.), The Sociology of Job Training (Research in the Sociology of Work, Volume 12) (pp. 197 - 227). Emerald Group Publishing Limited.

Phelps, A. F., \& Horman, M. J. (2010). Ethnographic theory-building research in construction. Journal of Construction Engineering and Management, 136(1), 58-65.

Pink, S., Tutt, D., Dainty, A., \& Gibb, A. (2010). Ethnographic methodologies for construction research: knowing, practice and interventions. Building Research \& Information, 38(6), 647-659.

Rawlinson, F. and Farrell, P. (2009) The vision of zero risk tolerance in craft workers and operatives: an unattainable goal?, in Dainty, A.R.J. (ed.) Proceedings of the 25th Annual ARCOM Conference, Association of Researchers in Construction Management, Reading, pp. 1203-12

Reason, J. (2008). The Human Contribution: unsafe acts, accidents and heroic recoveries. Surrey: Ashgate Publishing Company.

Rozenfeld, O., Sacks, R., Rosenfeld, Y., \& Baum, H. (2010). Construction job safety analysis. Safety science, 48(4), 491-498.

Sanne, J. M. (2008). Incident reporting or storytelling? Competing schemes in a safety-critical and hazardous work setting. Safety Science, 46(8), 1205-1222.

Smith, S., Sherratt, F., \& Oswald, D. (2017) The antecedents and development of unsafety, Proceedings of ICE - Management, Procurement and Law, 170(2), 59-67

Silverman, D. (2013). Doing qualitative research: A practical handbook. SAGE Publications Limited.

Strauch, B. (2015). Can we examine safety culture in accident investigations, or should we? Safety Science, $77,102-111$.

Walker, G. W. (2010). A safety counterculture challenge to a "safety climate". Safety Science, 48(3), 333-341 